\section{Expression of ERK1 and ERK2 in prostate cancer}

\author{
Norelia Torrealba, ${ }^{1}$ Benito Fraile, ${ }^{1}$ \\ Gabriel Olmedilla,2 \\ Pilar Martínez-Onsurbe,2 \\ Manuel Guil-Cid,3 Ricardo Paniagua, 1 \\ Mar Royuela1 \\ 1Department of Biomedicine and \\ Biotechnology, University of Alcalá, \\ Madrid; 2Department of Pathology, \\ Príncipe de Asturias Hospital, Alcalá de \\ Henares, Madrid; ${ }^{3}$ Department of \\ Urology, Príncipe de Asturias Hospital, \\ Alcalá de Henares, Madrid, Spain
}

\begin{abstract}
Prostate cancer may emerge as result of dysregulated balance between cell proliferation and death rates, increased angiogenesis and chronic. These processes are regulated by numerous signaling proteins, including the mitogen-activated protein kinases (MAPKs). JNK, p38 and extracellular signal-regulated kinase (ERK) are the three major sub-families of MAPKs. The pro-oncogenic effects of ERK isoforms (ERK1 and ERK2) lie in their aberrant activation through phosphorylation by any mutation along the pathway of receptor tyrosine kinase (RTK)-Ras-Raf-MEK-ERK1/2. Once activated, ERKs phosphorylate cytoskeletal proteins, kinases, and transcription factors. Active ERK proteins induce strong proliferative and anti-apoptotic effects. Our group has tested variations in expression, activation and localization of ERKs in human prostate. Differential ERK1/2 expression and phosphorylation status may be linked to the progression of prostate cancer. The major striking observation is that ERKs are expressed in tumors with higher proportion than normal prostate. We believe that this is an important notion because the status (expression, localization, phosphorylation and the ERK1/ERK2 ratio) of ERK in the prostate may be developed into an important prognostic marker that predicts patient responce to the anti-cancer treatment.
\end{abstract}

\section{Introduction}

Prostate cancer is the most common cancer among men worldwide, and is one of the most common causes of cancer-related deaths. It is the second most common cancer diagnosed after lung cancer. ${ }^{1}$

The prostate is an accessory male reproductive structure that surrounds the urethra into the pelvic cavity. The function of the prostate is to provide a series of compounds to ejaculate. Human prostate tissue is divided into two components: glands and stroma. Both are separated by a basement membrane. ${ }^{2}$ The glands are made of two cell types: basal cells and secretory cells. Another cell type can be also observed by the means of special chromogramin A or B statining. ${ }^{2}$

Prostate cancer (PC) is characterized by slow-growing malignancy associated with a multifocal origin. The majority of these tumors are diagnosed as adenocarcinomas, with its origin in the prostate glands. Prostate tumors grow multifocally in the prostate and rarely produce macroscopic tumor nodules. ${ }^{4}$ Tumor origin seems to be the secretory cells ${ }^{3}$ although some studies suggest it could also be initiated from basal cells. ${ }^{4}$ However, the pathological diagnosis is based on the absence of basal cells. ${ }^{3,4}$

The normal adult prostate is in a homeostatic state, as the result of the balance between the rates of proliferation and cell death. The abnormal growth occurs when the proliferation rate exceeds the rate of cell death, or the death rate falls below the rate of cell proliferation. ${ }^{5}$ When the pro-apoptotic pathways are inhibited, the cells can proliferate almost continuously. Such cells lose ability to cease cell cycle, to repair their DNA, or to commit suicide if the DNA damage is not repaired.

Studies on histological-molecular aspects that predict, identify and extend our knowledge about the factors associated with the progression of PC are in high demand. Currently, prostate-specific antigen (PSA), a protein produced by cells of the prostate gland and measured in patient blood sample, serves as high sensitivity organ-specific marker. However, PSA is low specificity marker and thus results in a failure to identify the disease in a significant proportion of patients. ${ }^{6}$ Measurements of other proteins may improve diagnostic specificity for early detection and more accurate grading of PC using the Gleason scale. The Gleason grading system assigns PC a score from 1 to 10 based upon the microscopic appearance of tumor cells. Cancers with a higher Gleason score are less differentiated, more aggressive (tending to form local and distant metastases) and have a worse prognosis for the patient survival rate. Tumors with Gleason scores 8-10 tend to be advanced neoplasms that are unlikely to be cured.

In recent years we have been monitoring variations in expression, activation and localization of MAPKs. ${ }^{7}$ We used samples from human prostate, obtained by radical prostatectomy, transurethral resection and autopsies. We have correlated the results of immunohistochemistry with clinical data of patients, such as PSA levels (pre- and postoperative), Gleason score, presence of metastases, surgical margin
Correspondence: Mar Royuela, Department of Biomedicine and Biotechnology, University of Alcalá, E-28871 Alcalá de Henares (Madrid), Spain.

Fax: +34.91.8854799

E-mail:mar.royuela@uah.es

Key words: MAPKs; ERK1; ERK2; p-ERK and prostate cancer.

Acknowledgments/Funding: this work was supported by grants from the Instituto de Salud Carlos III (PI13/01801) of Spain.

Contributions: MR designed the study and carried out the immunohistochemistry and statistical studies; NT created the figures; PM, G0, BF and MG prepared and provided the tumors biological samples and participated in the immunohistochemistry studies; NT and MR edited the paper and commented on the interpretation of the results; MR and RP participated in the study coordination and supervision. All authors read, discussed and approved the final manuscript.

Conflict of interest: the authors declare that they have no conflict interest.

Received for publication: 12 May 2015.

Revision received: 27 November 2015.

Accepted for publication: 27 November 2015.

This work is licensed under a Creative Commons Attribution NonCommercial 3.0 License (CC BYNC 3.0).

(C) Copyright N. Torrealba et al., 2015

Licensee PAGEPress, Italy

MAP Kinase 2015; 4:5265

doi:10.4081/mk.2015.5265

status, cancer recurrence, and patient survival at five years after prostatectomy (surgical removal of prostate).

Positive surgical margin is defined as the presence of tumor extending through the tinted area that represents the place where the urologist has cut to remove the tumor. ${ }^{8}$ Eastham et al. ${ }^{8}$ described positive surgical margins between $10 \%$ and $48 \%$ of patients treated with radical prostatectomy. A positive surgical margin has adverse impact on the probability of progression-free survival, both locally and biochemical, and the development of metastases after radical prostatectomy in multivariate analyzes. ${ }^{9}$ The risk of biochemical progression with positive surgical margin rate has a 1.5 to 2.6 compared to a negative margin. ${ }^{8}$ However, most studies suggest that only a third of patients with positive surgical margin have biochemical progression. ${ }^{9}$

The androgen deprivation therapy is the first-line treatment for metastatic prostate cancer. ${ }^{10}$ Decreasing androgen causes tumor regression. Although metastatic patients ini- 
tially have a good response to treatment, in almost all patients there is an increase in circulating levels of androgens within 2-3 years. Some patients develop castration resistance. ${ }^{10}$ Therefore, understanding the processes that render prostate cancer cell resistant to androgen deprivation therapy is a major challenge. Accordingly, our research is aimed at identifying prognostic markers that may predict possible relapses, development of hormone resistant prostate cancer and, thus find some prognostic factors after radical prostatectomy for preventing early recurrences.

The cell has three similar signaling pathways called mitogen-activated protein kinase (MAPKs) cascades. MAPK pathways are responsible for transmitting extracellular signals evoked by peptide growth factors, hormones, cytokines or various kinds of stress. ${ }^{11}$ They play important roles in the regulation of cell growth, differentiation, survival, apoptosis, and inflammatory stress. The three major subfamilies of MAPKs are JNK (c-Jun NH2-terminal kinase or SAPK), p38 (CSBP) and ERK (extracellular signal-regulated kinase).12

JNKs are commonly associated with apoptosis induction, while ERKs are associated with mitogenesis and inversely related to apoptosis. In most biological systems ERKs are associated with proliferation, but in some cases sustained activation of ERK induces cell death or have anti-proliferative effects. ${ }^{13}$ Multiple anti-cancer drugs are seen to induce sustained ERK signaling, as pemetrexed in lung cancer, paclitaxel in esophageal squamous cancer or cisplatin in many different cancer cells. ${ }^{14}$ Contradictory effects on cell death have been ascribed to p38.13 While ERK1/2 has been attributed a prominently protumoral role, the activation of JNK and p38-MAPK can exert both pro- and anti-tumor effects, which seems to depend on the cell type, stimulus, intensity and duration of the signal, and the interaction with other signaling pathways. Several studies have recently revealed the involvement of MAPKs in cancer associated with inflammation. The chronic inflammation is related to the possibility of developing prostate cancer.11,13-16

Among the signaling pathways most frequently dysregulated in human cancer is the RTK-RAS-RAF-MEK-extracellular signal-regulated kinase 1 and 2 (ERK1/2) pathway.13,14 In recent years much research has focused on the development of new inhibitors signaling molecules associated with this pathway. ${ }^{15,16}$

ERK1 and ERK2 are involved in the processes triggered by the arrival of mitogenic growth factors and controls in turn cell proliferation and differentiation. ${ }^{14}$ ERK1 and ERK2 are activated when the sequence TEY at their activation loop is dually phosphorylated by MEK1/2 kinases, while located in cytoplasm. ${ }^{14}$

The RAS/MAPK cascade is activated by a variety of growth factors, hormones and cytokines. Upon binding to receptor tyrosine kinases (RTKs), such as epidermal growth factor receptor (EGFR) or transforming growth factor receptor I and II (TGFBRI-TGFBRII). These ligands trigger receptor dimerization and autophosphorylation on the tyrosine residues in the $\mathrm{C}$-terminal region, which provides docking sited for adapter proteins SHC (Src homology 2 domain containing transforming protein 1) and GRB2 (growth factor receptor-bound protein 2). GRB2 recruits the cytosolic guanine nucleotide exchange factor SOS (Son of Sevenless) to the plasma membrane. Membrane recruitment is sufficient to activate SOS that in turn activates the membranebound small GTPase RAS by catalyzing the exchange of GDP with GTP.14-17 Then, RAS phosphorylates RAF1. In this way, a MAPK cascade is initiated in which RAF1 phosphorylate sequentially MEK1/2 and ERK1/2. Later, ERK1/2 translocate to the nucleus in a process that culminates in modulation of gene transcription through the activation of several transcription factors such as ETS1, ATF-2, cFOS, c-MYC, ELK1 or NF-kB.18 At the same time, it can also phosphorylate cytoplasmic and nuclear kinases, such as MNK1, MNK2, MPKAP2, RSK or MSK1.19 ERK1/2 are an important checkpoint protein, whose persistent activation may trigger the development of malignancies and play a fundamental role in cancer metastasis. ${ }^{14,16,20}$

At present, the inhibitors of the kinase function of RAF and MEK represent the most studied and advanced approaches for blocking ERK signaling. There are several inhibitors under evaluation but there are not many inhibitors that directly target ERK. The published data is discussed. ${ }^{14,21}$

\section{Materials and Methods}

All the procedures were examined and approved by the University of Alcalá and Principe de Asturias Hospital Ethics Committees (PI13/1801; 2013/003/20130214) and were in accordance with the ethical standards of the Committee for Human Experimentation, with the Helsinki Declaration of 1975 (revised in Tokyo 2004) and the Committee on Publication Ethics guidelines. This study was performed with the written consent of the patients or their relatives. All pathological, clinical or personal data were anonymized and separated from any personal identifiers. The present study included: i) transurethral resection or radical prostatectomies from 86 men (aged from 52 to 74 years) with PC (Table 1); and ii) histologically normal prostates (NP) obtained at autopsy (8-10 h after death) from 10 men (aged from 20 to 38 years) without histories or reproductive, endocrine or related diseases.

Immnohistochemical study has been performed as shown in previous publications. ${ }^{22,23}$ The primary antibodies used were: rabbit antihuman ERK-1; mouse anti-human ERK-2 and p-ERK (Santa Cruz Biotechnology, CA, USA). The immunostaining expression is the presence or absence of staining in prostate tissue section under study. It is negative when immunohistochemical staining is absent, and positive when staining is observed in the sample. In immunostaining intensity the stain coloration ranging from light pink to deep purple and very classified crosses (+) is observed. In the tumor, the glandular component (acinar and duct) cells are counted with the help of a hematology analyzer by differences according to color staining: weak $(1+)$, moderate $(2+)$ intense (3+) and strong (4+).

In stroma cells are observed microscopically counting the same fields as the tumor and shown as values of percentages. Then both cases are classified depending on the result: Negative (when no staining was observed), low positive, when the result is a cross (1+) and Positive results in high when the crosses are two or more crossings (2+ or greater). These results are used for statistical calculation. The main outcome measure of the study was time to biochemical progression at 10 years, defined as the time between definitive therapy to the first of at least two consecutive elevations in the total serum PSA level above $0.2 \mathrm{ng} / \mathrm{mL}$. Established prognostic variables

Table 1. Clinicopathological features of patients. Median (range)

\begin{tabular}{lc} 
Age (years) & $66.00(52-74)$ \\
Preoperative serum PSA (ng/mL) & $11.35(1.4-33)$ \\
\hline & $\%(n)$
\end{tabular}

$\begin{array}{cc}\begin{array}{l}\text { Preoperative serum PSA (ng/mL) } \\ \quad<10 \mathrm{ng} / \mathrm{mL}\end{array} & 36.0(31) \\ \quad \geq 10 \mathrm{ng} / \mathrm{mL} & 64.0(55) \\ \text { Gleason score } & \\ <7 & 82.6(71) \\ 7 & 9.3(8) \\ >7 & 8.1(7)\end{array}$

Clinical T stage

$\begin{array}{ll}\text { I } & 54.7(47) \\ \text { II } & 45.3(39)\end{array}$

\begin{tabular}{lc} 
Pathological T stage & \\
II & $65.1(56)$ \\
III & $31.4(27)$ \\
IV & $3.5(3)$ \\
\hline Perineural invasion (yes) & $26.7(23)$ \\
Positive surgical margins & $38.4(33)$ \\
\hline Node involvement (yes) & $4.7(4)$ \\
Biochemical progression (yes) & $33.7(29)$ \\
\hline Survival (yes) & $73.3(63)$ \\
\hline
\end{tabular}


included in the study were preoperative serum PSA levels, pathological and clinical T stages, postoperative Gleason score, perineural invasion, lymph node involvement, surgical margin status and overall survival. To evaluate the association between clinicopathological and immunohistochemical variables Spearman's test was performed. Log-rank test and KaplanMeier curves were used for survival comparisons. To explore the correlation of the studied immunohistochemical parameters and the established prognostic variables with biochemical progression, univariate and multivariate Cox proportional hazard regression analyses were performed. All statistical analyse ${ }^{23}$ were performed using the SPSS 22.0 software (SPSS Inc. Chicago, IL, USA). P values $<0.05$ were considered as significant.

\section{Results and Discussion}

According to immunostaining data, that our group has previously published ${ }^{22}$ ERK1 was detected in the cytoplasm of epithelial cells in the $90 \%$ of the samples obtained from PC patients (Figure 1A), which was similar to that obtained from normal prostate (Figure 1B) glands (94\%). ${ }^{22}$ In PC samples, ERK1 optical densities were increased with Gleason scores. ERK2 was detected in cytoplasm of the epithelial cells in $100 \%$ of samples obtained from normal prostate (Figure 1D) while in PC group (Figure 1C) appeared in $68 \%$ of patients.22 Optical density was the highest in PC, but no differences between Gleason groups. The pERK was found in the cytoplasm in $40 \%$ of normal prostate (Figure 1F) and 30\% of PC samples. $^{22}$ The highest optical density was observed in PC (Figure 2E) but no differences were found in Gleason score. ${ }^{22}$ Several authors showed than ERK is important in the progression of the malignancy in prostate cancer. We saw in our patients with PC a significant expression of ERK, perhaps related with cancer progression. Imada et al. ${ }^{24}$ described the biochemical relevance of ERK to the progression of PC, while according to our results Gioeli et al. ${ }^{25}$ found the correlation between the increase in ERK activation and advanced PC grade and stage. The normal prostate is composed of glands and stroma. We analyzed the differences of ERK expression in the prostatic stromal and tumor cells. The presence of ERK was indicated as positive result, whereas absence of antibody reactivity with ERK1, ERK2 or their phosphorylated forms (p-ERK) was considered as negative result (Table 2 Expression). Then, the staining intensities of ERK1, ERK2 and p-ERK were evaluated between stroma and tumor locations (Table 2 Intensity).

We see as ERK1 is expressed in tumor with
Table 2. Distribution of ERK-1, ERK-2 and p-ERK in PC samples (86) based on immunostaining score for expression and intensity.

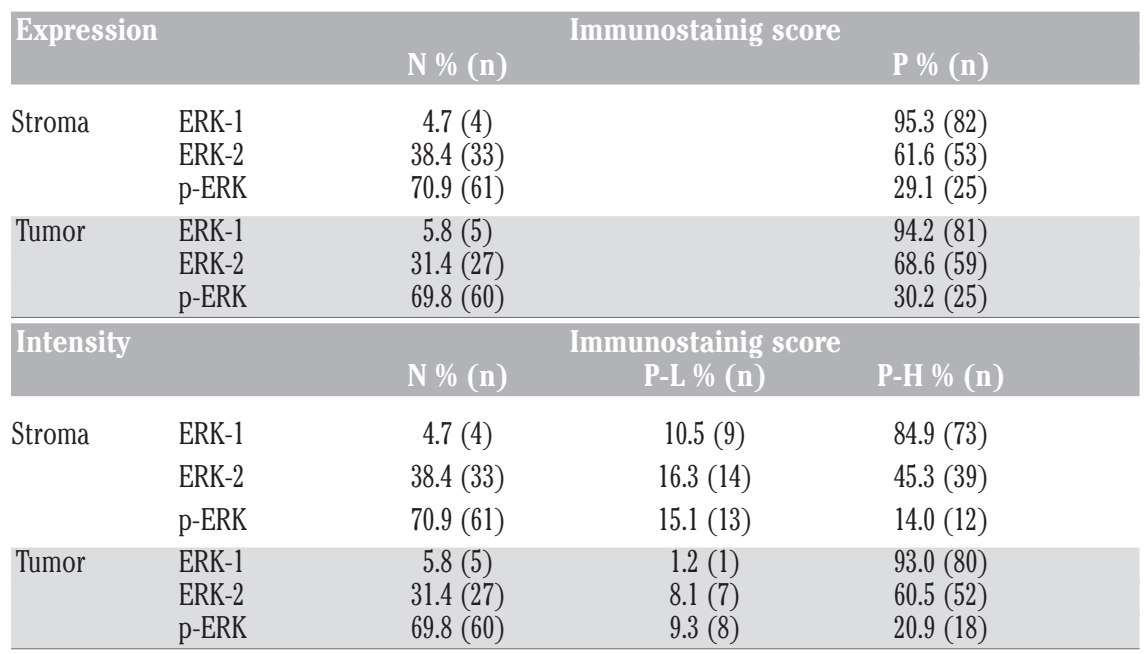

N, negative; P-L, Positive-low; P-H, Positive-high. Modified by Rodriguez-Berriguete et al., 2010.22
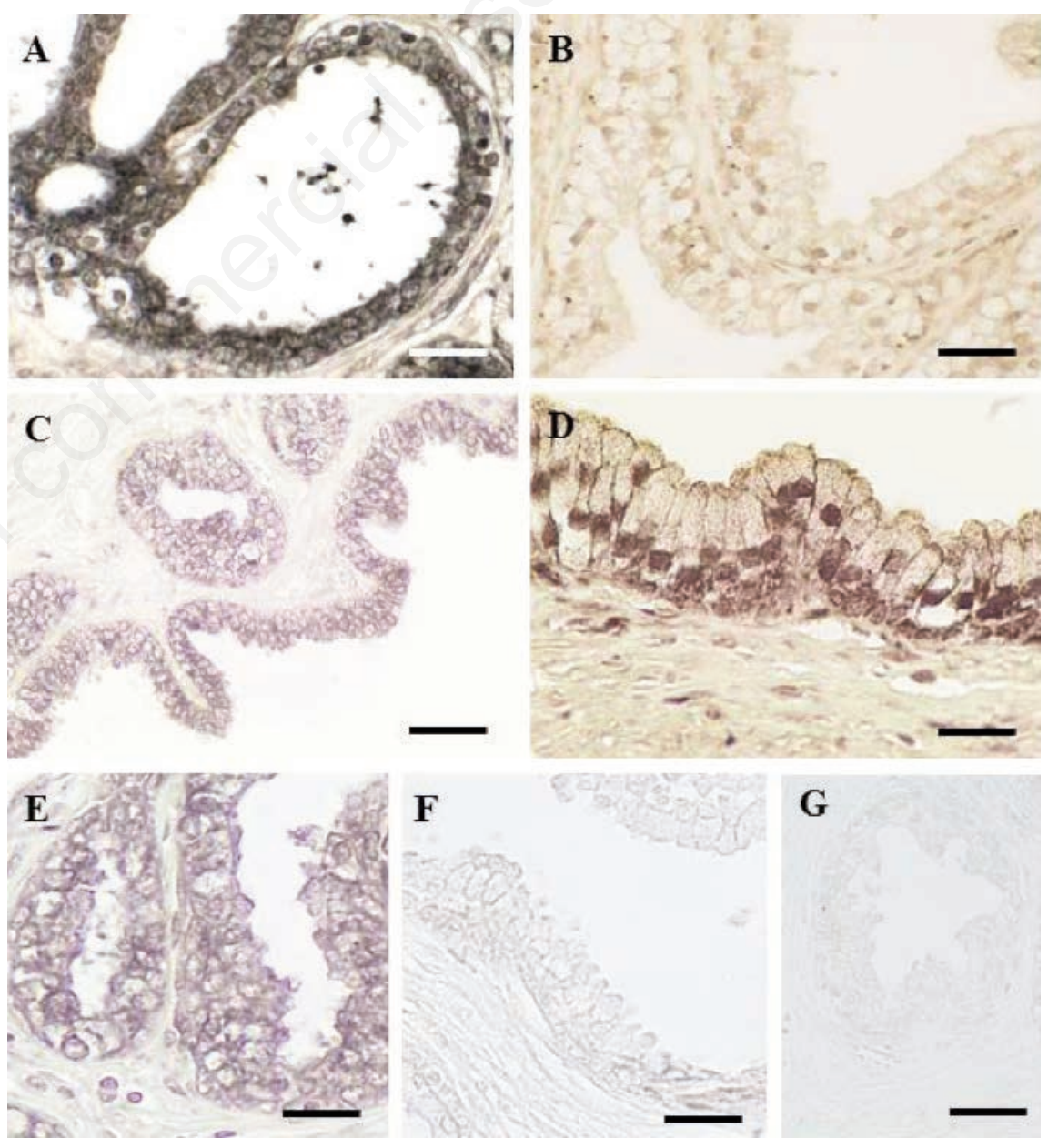

F

G

Figure 1. ERK-1 (A, B) ERK2 (C, D), p-ERK (E, F) and negative control (G) in cancer $(A, C, E)$ and normal prostate $(B, D, F, G)$. Scale bars: $30 \mu \mathrm{m}(C, G), 25 \mu \mathrm{m}(A), 20 \mu \mathrm{m}$ $(\mathrm{B}, \mathrm{E}, \mathrm{F})$ and $15 \mu \mathrm{m}(\mathrm{D})$. 
higher proportion than ERK2 and p-ERK. Also, we see the intensity in tumor for ERK1 has a higher proportion to ERK2 and p-ERK. For the stroma, the proportions are similar with those described above. Several authors have described as p-ERK is significantly decreased in PC. The authors see that in PC3 and DU145 cells (hormonal independent advanced prostate cancer cells) levels of expression for the members RAS/RAF/MEK/ERK cascades are low. The deletion of PTEN gene in PC could play a role in the suppression of this pathway. ${ }^{26}$

In this analysis, $r$ is the correlation coefficient and $\mathrm{P}$ marks a statistical significance. The correlation coefficient may range from -1 to 1 , where -1 or 1 indicates a perfect relationship. The further the coefficient is from 0 , regardless of whether it is positive or negative, the stronger the relationship between the two variables. Therefore, a coefficient of -0.213 is almost as strong as a coefficient of 0.241. Positive coefficients tell us that there is a direct relationship: when one variable increases, the other increases. Negative coefficients tell us that there is an inverse relationship: when one variable increases, the other one decreases.

ERK1 expression in stroma has indirect relation with nodal involvement (Table 3 Stroma) and with positive margins surgical in stromal intensity (Table 3 - Stroma). ERK activation is correlated with increasing of Gleason score, and these patients have a malignancy progression. ${ }^{26,27}$ Uzgare et al. ${ }^{27}$ and Junttila et $a l .{ }^{18}$ demonstrated in TRAMP (transgenic adenocarcinoma of the mouse prostate), that ERK activation has effects in prostatic epithelial cells, and it produces the tumor. ERK has differentially expressed and activated during prostate cancer progression. These authors think that the inactivation of ERK has a relationship with the emergence of a poorly differentiated metastatic and androgen-independent phenotype. Koul et al. ${ }^{28}$ showed in PC3 cell line that ERK signal transduction pathway has essential paper for cell migration, invasion and clonogenic activity. This clonogenic activity causes cells with the capacity to proliferate a colony of genetically identical cells.

In our patients, p-ERK is related in a direct manner with pathological T stage and indirectly with the survival of patients in the case of the expression in the stroma (Table 3 Stroma) and epithelium (Table 3 - Tumor). Intensity in tumor patients also stores relationships with the presence of p-ERK in the same manner (Table 3 - Tumor).

After evaluating the correlation of our MAP kinase, we studied the survival curves of Kaplan-Meier. Survival curves for classic markers confirmed the forecast for biochemical recurrence, $\mathrm{T}$ value pathological stage $(\mathrm{P}=0.051)$, Gleason score $(\mathrm{P}=0.003)$ and lymph node metastasis $(\mathrm{P}=0.000)$ but not for preoperative serum PSA and perineural invasion, positive surgical margins. ${ }^{23}$
The regression model for univariate Cox proportional hazards were used to study the association between each variable separately study the event of interest (time of biochemical recurrence) providing hazard ratio (Hazard ratio) which estimates differences in risk of experiencing the effect of interest between different groups of patients. It also allows us to corroborate the results of the Kaplan-Meier.23 In this review we have added charts KaplanMeier classical markers to demonstrate that the values of Log rank are corroborated in the study of univariate Cox provided contrary to the values obtained in the survival curves of cytokines study showing no significant values. ${ }^{23}$

We performed a Univariate Cox analysis of classical markers; also we performed analysis for biochemical recurrence. ${ }^{23}$ We note that the pathological T stage, Gleason score and node involved relate to biochemical recurrence-free time (Table 4), thus fulfilling our patient group some classical predictions.

We performed survival curves of KaplanMeier for the cytokines of the study and we evaluated the univariate Cox. In this group of patients, we did not obtained significant results for any cytokines. We also did not find data in this regard in the literature.

Ricote et al. ${ }^{29}$ demonstrated that p-ERK has an important role in the increase of proliferation in LNCaP cells; in this study p-ERK was notably increased by TNF- $\alpha$.

Table 3. Correlation between immunohistochemical variables and clinicopathological features for expression and intensity.

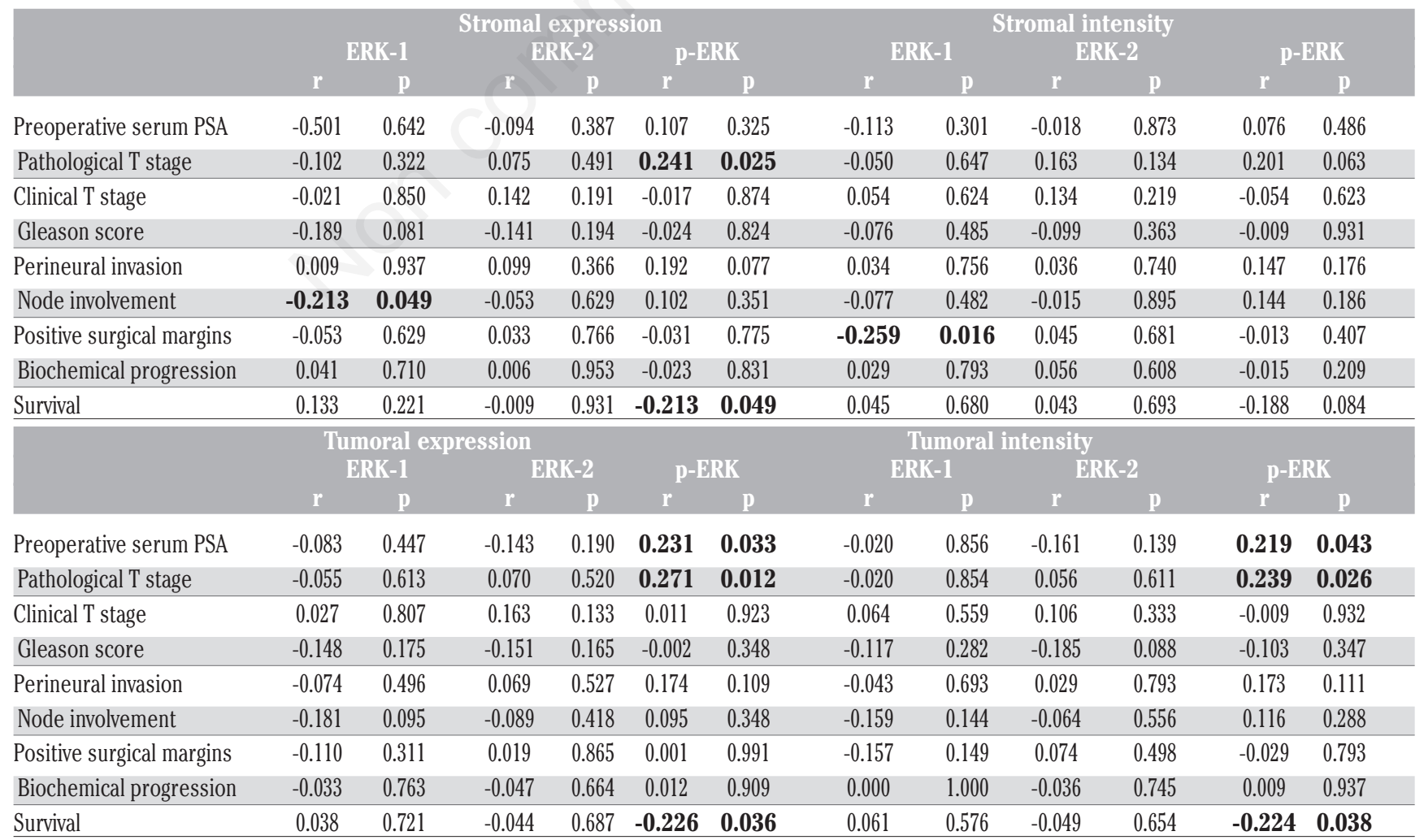

Bold values indicate significant correlations. 
Table 4. Univariate Cox proportional hazard regression analysis for biochemical progression according to classical markers.

\begin{tabular}{|c|c|c|}
\hline & P value & Hazard ratio $(95 \% \mathrm{CI})$ \\
\hline Preoperative serum PSA & 0.146 & $1.879(0.802-4.401)$ \\
\hline Pathological T stage & 0.017 & $2.017(1.132-3.595)$ \\
\hline Gleason score & 0.000 & $2.966(1.818-4.838)$ \\
\hline Perineural invasion & 0.077 & $1.971(0.930-4.177)$ \\
\hline Node involvement & 0.001 & $6.166(2.068-18.318)$ \\
\hline Positive surgical margins & 0.404 & $1.367(0.657-2.844)$ \\
\hline
\end{tabular}

Shimada et al. ${ }^{26}$ has been described the status of activated phosphorylated ERK in human prostate cancer cells with pathological parameters; ERK cannot be activated without being phosphorylated at the same time unless it is a mutant. They suggested that the activation of ERK signaling is related with prostate cancer progression. ERK is commonly considered as anti-apoptotic, cell growth-related kinase, and as such ERK activation favors clinical tumor progression. ERK is associated with malignancies and for this is investigated in several studies. In other tumors as breast, lung, colon and stomach, the role of ERK in the progression and tumor development also has been described. ${ }^{30,31}$

Our research group showed the important role of the IL6/ERK transduction pathway in the activation of NF-kB in these patients. 26,27 IL6/ERK transduction pathway stimulated the translocation of NF-kB to the nucleus in PC. $\mathrm{NF}-\mathrm{kB}$ in the nucleus promotes the expression of inhibitors of apoptosis proteins and BCL2, and this is relation with the malignance of the tumor.13,22,23 Our large experience makes us think that the use of these parameters could be a useful tool in clinic-pathological practice.

In the last decade, researchers have progressed in the knowledge of prostate cancer initiation, progression and biomarkers associated with progression of disease. Several studies associate ERK with a pro-tumor role. ERK is involved in several transduction pathways such as IL6/ERK/NF-kB related with advanced malignance and the molecular roles of ERK are not known at all. The aim of future studies might be directed in the knowledge of the different mechanisms that provoke pro-tumor effect of ERK, in order to finding effective therapeutic targets that could reduce the effect of ERK in prostate cancer.

\section{References}

1. Jemal A, Bray F, Center MM, et al. Global cancer statistics. CA Cancer J Clin 2011;61:69-90.
2. Cunha GR. Mesenchymal-epithelial interactions: past, present, and future. Differentiation 2008;76:578-86.

3. Xin L. Cells of origin for cancer: an updated view from prostate cancer. Oncogene 2013;32:3655-63.

4. Hanahan D, Weinberg RA. Hallmarks of cancer: the next generation. Cell 2011;144:646-74. Egevad LL. The 2005 International Society of Urological Pathology (ISUP) Consensus Conference on Gleason Grading of Prostatic Carcinoma. Am J Surg Pathol 2005;29:1228-42.

6. Roddam AW, Duffy MJ, Hamdy FC, et al. Use of prostate-specific antigen (PSA) isoforms for the detection of prostate cancer in men with a PSA level of $2-10 \mathrm{ng} / \mathrm{ml}$ : systematic review and meta-analysis. Eur Urol 2005;48:386-99.

7. Rodríguez-Berriguete G, Prieto A, Fraile B, et al. Relationship between IL-6/ERK and NF-KB: a study in normal and pathological human prostate gland. Eur Cytokine Netw 2010;21:241-50.

8. Eastham JA, Kuroiwa K, Ohori M, et al. Prognostic significance of location of positive margins in radical prostatectomy specimens. Urology 2007;70:965-9.

9. Swindle P, Eastham JA, Ohori M, et al. Do margins matter? The prognostic significance of positive surgical margins in radical prostatectomy specimens. J Urol 2005; 174:903-7.

10. Steinestel J, Schrader AJ, Luedeke M. Resistance to androgen-pathway drugs in prostate cancer. N Engl J Med 2014;371: 2234.

11. Kyriakis JM, Avruch J. Mammalian MAPK signal transduction pathways activated by stress and inflammation: a 10-year update. Physiol Rev 2012;92:689-737.

12. Frank SB, Miranti CK. Disruption of prostate epithelial differentiation pathways and prostate cancer development. Front Oncol 2013;3:273.

13. Rodriguez-Berriguete G, Fraile B, Martinez-Onsurbe $\mathrm{P}$, et al. MAP kinases
5. Epstein JI, Allsbrook WC Jr, Amin MB, and prostate cancer. J Signal Transduct 2012;2012:169170.

14. McCubrey JA, Steelman LS, Chappell WH, et al. Roles of the Raf/MEK/ERK pathway in cell growth, malignant transformation and drug resistance. Biochim Biophys Acta 2007;1773:1263-84.

15. Huang P, Han J, Hui L. MAPK signaling in inflammation-associated cancer development. Protein Cell 2010;1:218-26.

16. Frémin $\mathrm{C}$, Meloche $\mathrm{S}$. From basic research to clinical development of MEK1/2 inhibitors for cancer therapy. J Hematol Oncol 2010;3:8.

17. Zhang Y, Chen HX, Zhou SY, et al. Sp1 and c-Myc modulate drug resistance of leukemia stem cells by regulating survivin expression through the ERK-MSK MAPK signaling pathway. Mol Cancer 2015;14:56.

18. Junttila MR, Li SP, Westermarck J. Phosphatase-mediated crosstalk between MAPK signaling pathways in the regulation of cell survival. FASEB J 2008;22:95465 .

19. Zebisch A, Czernilofsky AP, Keri G, et al. Signaling through RAS-RAF-MEK-ERK: from basics to bedside. Curr Med Chem 2007;14:601-23.

20. Peng S, Zhang Y, Zhang J, et al. ERK in learning and memory: a review of recent research. Int J Mol Sci 2010;11:222-32.

21. Goetz EM, Ghandi M, Treacy DJ, et al. ERK mutations confer resistance to mitogenactivated protein kinase pathway inhibitors. Cancer Res 2014;74:7079-89.

22. Rodríguez-Berriguete G, Prieto A, Fraile B, et al. Relationship between IL-6/ERK and NF-KB: a study in normal and pathological human prostate gland. Eur Cytokine Netw 2010;21:241-50.

23. Rodríguez-Berriguete G, SánchezEspiridión B, Cansino JR, et al. Clinical significance of both tumor and stromal expression of components of the IL-1 and TNF- $\alpha$ signaling pathways in prostate cancer. Cytokine 2013;64:555-63.

24. Imada K, Shiota M, Kohashi K, et al. Mutual regulation between Raf/MEK/ERK signaling and Y-box-binding protein-1 promotes prostate cancer progression. Clin Cancer Res 2013;19:4638-50.

25. Gioeli S, Mandell JW, Petroni GR, et al. Activation of mitogen-activated protein kinase associated with prostate cancer progression. Cancer Res 1999;59:279-84.

26. Shimada K, Nakamura M, Ishida E, Konishi N. Molecular roles of MAP kinases and FADD phosphorylation in prostate cancer. Histol Histopathol 2006;21:415-22.

27. Uzgare AR, Kaplan PJ, Greenberg NM. Differential expression and/or activation of P38MAPK. Erk $1 / 2$ and jnk during the initation and progression of prostate cancer. Prostate 2003;55:128-39. 
28. Koul S, Huang M, Chaturvedi L, et al. p42/p44 Mitogen-activated protein kinase signal transduction pathway regulates interleukin-6 expression in PC3 cells, a line of hormone-refractory prostate cancer cells. Ann N Y Acad Sci 2004;1030:253-7.

29. Ricote M, García-Tuñón I, Fraile B, et al.
P38 MAPK protects against TNF-alpha-provoked apoptosis in LNCaP prostatic cancer cells. Apoptosis 2006;11:1969-75.

30. Royuela M, Arenas MI, Bethencourt FR, et al. Regulation of proliferation/apoptosis equilibrium by mitogen-activated protein kinases in normal, hyperplastic, and carci- nomatous human prostate. Hum Pathol 2002;33:299-306.

31. Tahir AA, Sani NF, Murad NA, et al. Combined ginger extract \& Gelam honey modulate Ras/ERK and PI3K/AKT pathway genes in colon cancer HT29 cells. Nutr J 2015;14:31. 